\title{
A ZigBee-based Building Energy and Environment Monitoring System Integrated with Campus GIS
}

\author{
Kun Qian ${ }^{1,2}$, Xudong Ma ${ }^{1,2}$, Changhai Peng ${ }^{3,4,5}$, Qing Ju ${ }^{1,2}$ and Mengyuan Xu ${ }^{1,2}$ \\ ${ }^{1}$ Key Lab of Measurement and Control of Complex Systems of Engineering, Ministry \\ of Education, P R China \\ ${ }^{2}$ School of Automation, Southeast University, Nanjing 210096, PR China \\ ${ }^{3}$ School of Architecture, Southeast University, Nanjing 210096, PR China \\ ${ }^{4}$ IIUSE, Southeast University, Nanjing 210096, PR China \\ ${ }^{5}$ Key Laboratory of Urban and Architectural Heritage Conservation, Ministry of \\ Education, P R China \\ kqian@seu.edu.cn \\ Abstract
}

In this paper, the design and development of a Building Energy and Environment Monitoring System (BEEMS) for smart campus applications is proposed. The system is implemented based on distributed sensor nodes using ZigBee technology, which empowers the collection and monitoring of various ypes of measurements that reflect the energy consumption and environmental status of buildings. These parameters include temperature, humidity, air velocity, sound, bubble globe temperature, TVOC, CO2 concentration, air quality level, et al. The application gofware system is developed using jQuery-based Ajax general interactive architecture, and further integrated with campus GIS, which offers rich analysis and report functions for monitoring both energy consumption and environment parameters.

Keywords: Built ênvironment, Monitoring system, ZigBee Network, Energy consumption

\section{Introduction}

In smart campus applications, an appraisal of built energy and environment provides sufficient evidence to the understanding of the impact of ambient surroundings on human health and thermal comfort. In order to make a valid assessment and mitigate the burden of manual monitoring, automatic environmental measures and monitoring systems are attracting increasing atténtion.

The earliest environmental measures and monitoring systems for smart buildings made use of eabled communication technology, such as CAN-bus, Power Line Carrier (PLC) [1]. However cabled communication systems were not very aesthetically pleasing solutions. ZigBee is a set of specifications created specifically for wireless sensor networks (WSNs)[1], [3]. It was built on IEEE 802.15.4 standard, which restricts the data rate to $250 \mathrm{kbps}$ in the global 2.4-GHz Industrial, Scientific, Medical (ISM) band, while also specifying low power consumption and cost. Zigbee-based sensing systems require low latency, low data rates, low cost, and low energy consumption. As a result, residential and commercial applications [4] include lighting controls, smoke and $\mathrm{CO} 2$ detectors, home security, automatic utility meter readings, building environmental controls, et al. 
In this paper, a WSN based distributed monitoring system for building energy and environment monitoring is proposed. ZigBee technology is introduced to environment monitoring and integrated into a Building Energy and Environment Management System (BEEMS), in which energy consumption monitoring using traditional electric meters and environment parameters monitoring. The utilization of ZigBee WSNs outperforms traditional techniques in providing a low power-cost and fully distributed building environment monitoring methodology. Moreover, the system is featured with the integration with campus Geographic Information System (GIS), which improves the user experience by providing easy access to different scale of campus, e.g., buildings, floors and rooms.

\section{System Development}

\subsection{System Overview}

The BEEMS is developed with a Browser/Server structure as shown in Figure 1 . In the BEEMS system, two groups of field sensors are deployed. The first groy $p$ is regular digital meters that capture consumption of energy, such as electric, gas, et at A smart gateway collects data from these meters on LONWORKS fieldbus and connects to the control network of the building. The second group is distributed wireless sensors deployed in different rooms that measure environmental parameters. A base station is deployed that gathers and stores environmental parameters measured in this building, and it's farther connected to the control network of the building and the campus network



Figure 1. The BEEMS System Architecture

\subsection{Deployment of Wireless Sensor Nodes}

For this implementation, Sensor Nodes are made up of Mote Processor/Radio Platforms (M) and Sensors (S). Each node is a fully integrated, rugged sensor package that uses energyefficient radio and sensors for extended battery-life and performance. Each node integrates 
Crossbow's IRIS/IMOTE2 family processor/radio board and antenna that are powered by rechargeable batteries. The node is capable of radio range of $10 \mathrm{~m}$ to $80 \mathrm{~m}$ (indoor) and $50 \mathrm{~m}$ to $300 \mathrm{~m}$ (outdoor) depending on deployment, remembering that the nodes themselves form a wireless mesh network and to extend the range of coverage simply adding additional wireless mesh networks expands the range of coverage. The nodes come pre-programmed and configured with Crossbow's XMesh low-power networking protocol. Figure 2 shows the scheme of the WSNs.

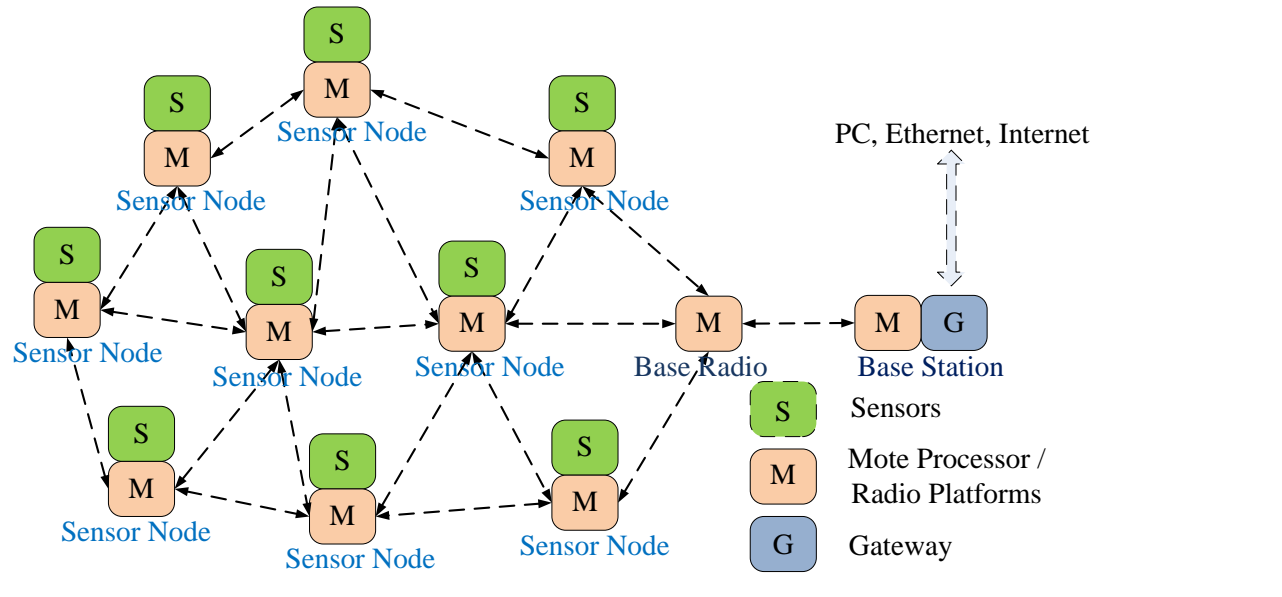

Figure 2. Scheme of WSNs

Figure 3 shows the list of hardware modules usêd in the system:

- Q120, an embedded Sensor Network gateway device. The gateway runs the Debian Linux operating system and comes preloaded with Crossbow's Sensor Network management and data visualization software packages, eKoView and XServe.

- MIB520CB an USB wireless interface board and programming interface. It provides USB connectivity to the IRIS and MICA family of Motes for commun cation and in-system programming.

- XM2110CB, wireless radio interface board. The XM2110CB is based on the Atmel ATmega 1281, a 1ow-power microcontroller which runs MoteWorks from its internal flash memory. The IRIS 51-pin expansion connector supports Analog Inputs, Digital I/Q, $2 \mathrm{C}$, SPI and UART interfaces.

- CITY iRcel-CO2, an environmental sensor. It offers Non-Dispersive Infra-Red (NDIR) for General Purpose Portable/Fixed CO2 Detection. Measurement Range is $0-5 \%$ vol. Carbon Dioxide; Accuracy $\left(-20^{\circ} \mathrm{C}\right.$ to $\left.+50^{\circ} \mathrm{C}\right)$ is within $\pm(0.1 \%$ vol $\mathrm{CO} 2+$ $4 \%$ of concentration); Response Time (t90)<35 Seconds.

PID-AH, an Alphasense Photo Ionisation Detector. Minimum detection level is 5 (ppb isobutylene); Linear range (3\% deviation) is 50 (ppm isobutylene); Overrange is 50 (ppm isobutylene); Sensitivity (linear range) $>20$ ( $\mathrm{mV} / \mathrm{ppm}$ isobutylene).

- EE66-VA3, an environmental sensor. It is designed for high accuracy measurement of lowest air velocities.

- JTC01, an environmental sensor. It is designed for high accuracy measurement of lowest air velocities.

- DEC1000, a measurement meter. It measures power energy consumption and sends the data via wireless communication. 


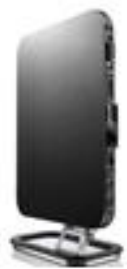

Q120

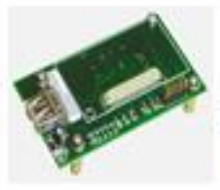

MIB520CB
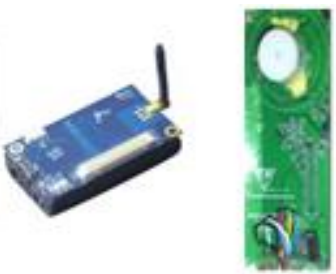

$\mathrm{XM} 2110 \mathrm{CB}$
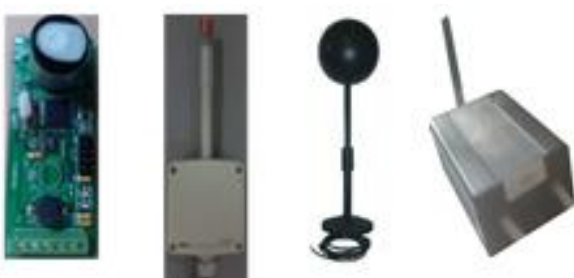

Figure 3. Sensor Nodes

\subsection{Application Software Development}

The monitoring application software follows a Brower/Server structure, which supports users to easily get access to both real-time and historic monitoring data yia the campus network or Internet. In the Web server, data is stored and managed in a SQL Server database. The application software is designed according to the Model-Vew-Controller (MVC) design pattern. In the MVC pattern, users send HTTP requests to the Controller via the browser; the controller selects adequate data from the Model and displays the results to the user through an proper View. In the design of Views, content, style and behavior are independently developed. In particular, the styles of web pages are formulated in the CCS lile and the interaction behaviors between web pages are implemented using jQuery [5]

\subsection{Database Design and Development}

The data table in the SQL server 2008 database designed according to the category of the statistical information that is compufed and different spatial scales of a campus, such as room level, floor level, building level and campus Yevel. Table 1 and Table 2 displays the data table designed for environmental monitoring parameters and energy monitoring parameters, respectively. The software ase LINQ to SQL as an interface to query data from the database. In addition, Ajax technique [6] is employed to implement the data interaction between client and server. Ajax provides ability of communicating with server asynchronously. For interchanging dat on the web, JavaScript Object Notation (JSON) [7] is used as a lightweight text-based open standard designed for human-readable data.

Table 1. Data Table for Environmental Monitoring Parameters

\begin{tabular}{llc} 
& environmentalClassID & className \\
\hline 2 & 00 & NULL \\
3 & 01 & Temperature \\
& Humidity \\
4 & 03 & Velocity of air flow \\
5 & 04 & Tadiation temperature \\
6 & 05 & Thermal comfort degree \\
7 & 06 & Atmospheric pressure \\
8 & 07 & CO2 \\
9 & 08 & TVOC \\
10 & 09 & O2 \\
11 & 10 & HCHO \\
12 & 11 & Illuminance \\
13 & 12 & Sound \\
\hline
\end{tabular}


Table 2. Data Table for Energy Monitoring Parameters

\begin{tabular}{ccc}
\hline & energyClassID & className \\
\hline 1 & 00 & NULL \\
2 & 01 & Electricity consumption \\
3 & 02 & Water consumption \\
4 & 03 & Air consumption \\
5 & 04 & Central heating consumption \\
6 & 05 & Central cooling consumption \\
7 & 06 & Other energy consumption \\
\hline
\end{tabular}

\section{Integration with Campus GIS and Applications}

The system is applied in the campus of southeast university for monitoring buildings distributed in the whole campus. A series of sensor nodes are equipped in some floprs of the monitored buildings. These nodes continuously sending messages of captured data to the server through the campus area network. Both energy and environmental measurements collected by the distributed sensing nodes are integrated in the application system for centralized display and management. Some typical functions of the monitoring application system are listed as follow.

- Energy Profile

Different categories of energy consumption are profiled based on the statistical information that is computed according to different spatial scales, such as room level, floor level, building level and campus level. The statistical data can also be reported and displayed in terms of table, chaptor bar figures according to different time scales of hour, day month and year. This important energy profiling function offers great supports for energy consumption analysis and making ene gy saving policies.

- Energy Comparisonand Ranking

Energy consumption statics conchuded in different categories and different items are compared and rânked explicitly at any time level. Hence it is obvious to find the unit with highest energy consumption.

- Data Analysis

The deviations of measured parameters to planned value can be displayed at any time of the day. Based on this, the tendency of energy consumption can be easily revealed. Moreover, the MaXMMin value of a measured parameter that reported at different time of the day can be analysis, so that the feature of such type of energy consume can be discovered.

The application software is further integrated with the $3^{\text {rd }}$ party three-dimensional map of the campuS known as Southeast University GIS (available at http://map.seu.edu.cn/ugis/). The integration uses ImageMapster1.2, an interactive map plug-in to web page and JavaScript. As a result, the application software provides friendly user's interface that enables easy access to different rooms, floors, buildings as well as campuses, as shown in Figure 4 and Figure 5. Figure 6 shows the result of querying the power consumption statistics (e.g., two rooms in which sensor nodes were installed), and the reports in bar chart format and line chart format, respectively. The application system has been tested in the university campus for several months and the system performance validates the reliability of the software. 

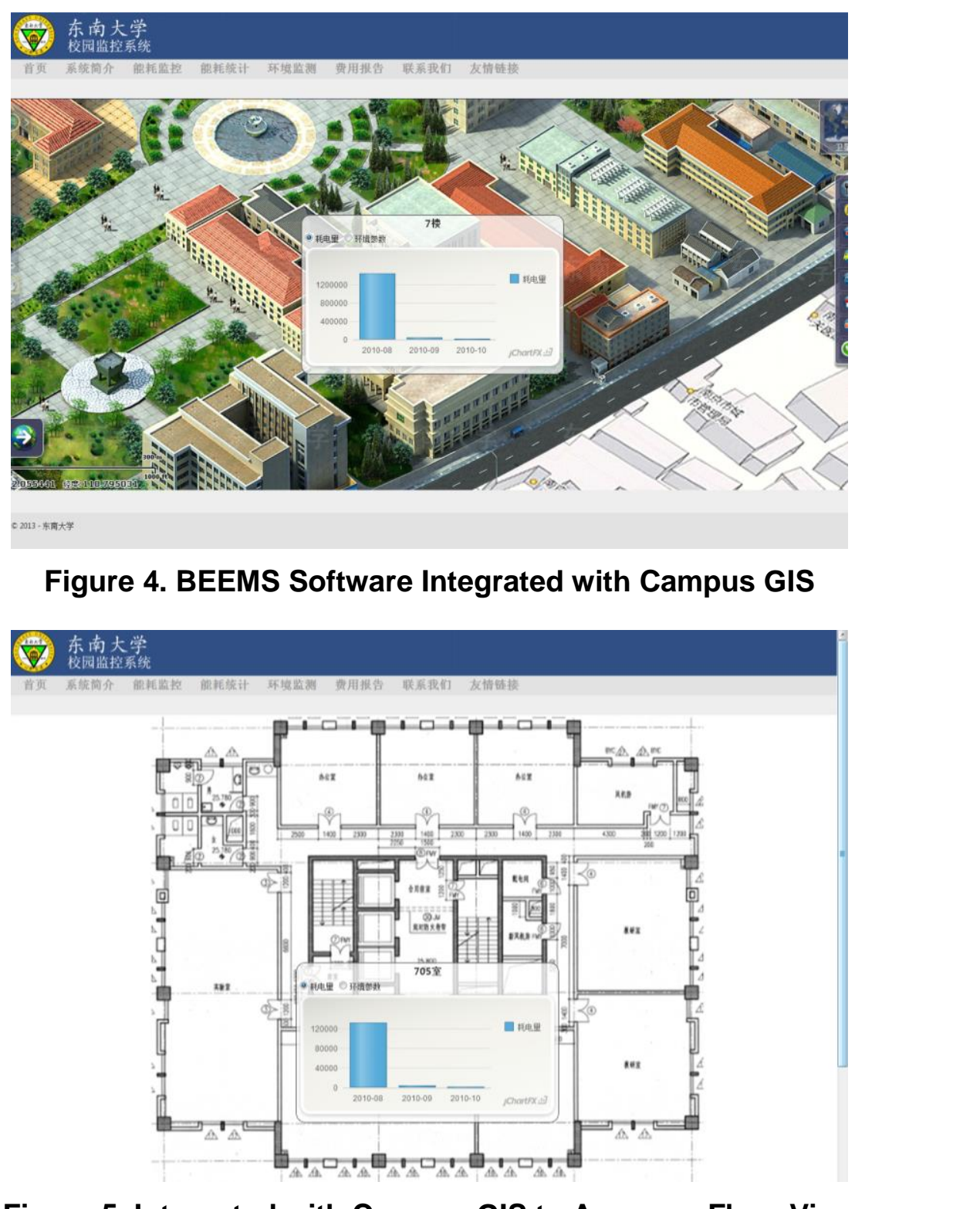

Figure-5. Integrated with Campus GIS to Access a Floor View



(a) Bar chart

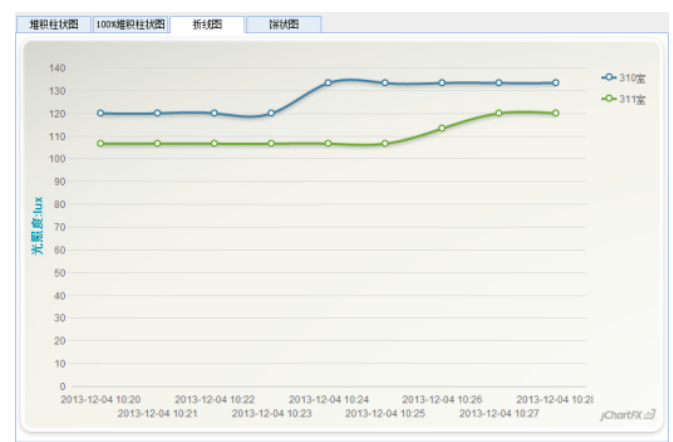

(b) Line chart 


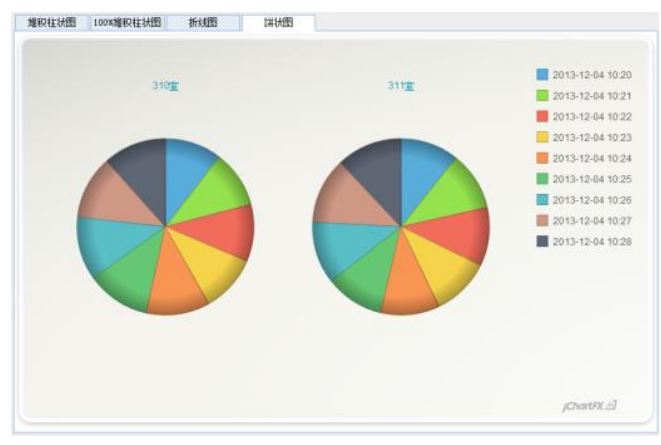

(c) Pie chart

\section{Figure 6. Power Consumption Statistical Results}

\section{Conclusion}

In this paper, the design and development of a Bulding Energy and Environment Monitoring System (BEEMS) for smart campus applications is proposed. The system is implemented based on distributed wireless sensor nodes, developed using ZigBee technology. Compared with other similar systems, the proposed system is featured by the integration of both energy and environment monitoring of buildings using Zigbee technology. Besides, the monitoring application software integrated with the campus o IS provides friendlier user experience. An application in a university campus yalidates the system performance in practical use. The system has provided a solution to the evaluation, monitoring and management of the energy and environment of large scale campus buildings.

\section{Acknowledgements}

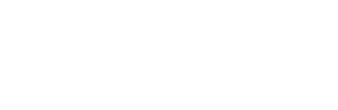

The work described in this paper is supported by the Key Program of Natural Science Foundation of Jiangsu Province (BK2010061), National Natural Science Foundation of China (51278107 and 61 105094), The R\&D Program of Ministry of Housing and Urban-Rural Development of People's Republic of China (2011-K1-2), the Open Project Program of the Key Laboratory of Urban and Architectural Heritage Conservation (Southeast University), Ministry of Education (KLUAHC1212), and National Key Technologies R\&D Program of China (2011BAJ03B04)

\section{References}

[1] L. C. Huang, H. C. Chang, C. C. Chen and C. C. Kuo, "A ZigBee-based monitoring and protection system for building electrical safety”, Energy and Buildings, vol. 43, no. 6, (2011), pp. 1418-1426.

[2] F. Baig, S. Beg and M. F. Khan, "Zigbee based home appliances controlling through spoken commands using handheld devices", International Journal of Smart Home, vol. 7, no. 1, (2013), pp.

[3] S J. Woo and B. D. Shin, "Efficient cluster organization method of zigbee nodes", International Journal of Smart Home, vol. 7, no. 3, (2013), pp. 45-56.

[4] M. H. Jin, C. J. Fu, C. H. Yu, H. R. Lai and M. W. Feng, "I BM Zigbee positioning method for smart home applications", International Journal of Smart Home, vol. 2, no. 2, (2008), pp. 127-139.

[5] Y. C. Jin, R. C. Wang, H. P. Huang and L. J. Sun, "Ubi-PowerMeter: A novel paradigm to reduce energy consumption”, International Journal of Smart Home, vol. 4, no. 2, (2010), pp. 11-30.

[6] JQuery, http://jquery.com

[7] Ajax, jQuery API Documentation, http://api.jquery.com/category/ajax/

[8] JSON, http://www.json.org/ 


\section{Authors}

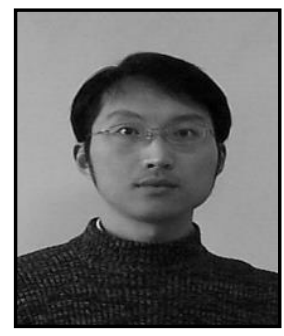

Dr. Kun Qian received the $\mathrm{Ph} . \mathrm{D}$. in control theory and control engineering from Southeast University in 2010. He is currently working as a lecturer at School of Automation, Southeast University. His research interests are intelligent robotic system and smart sensing.

Prof. Xudong Ma received the BS and MS degrees from Tsinghua University, P.R China, in 1985 and 1988, respectively. He is currently working as a Professor at the school of Automation, Southeast University. His research interests are realtime operating systems and intelligent robot control.

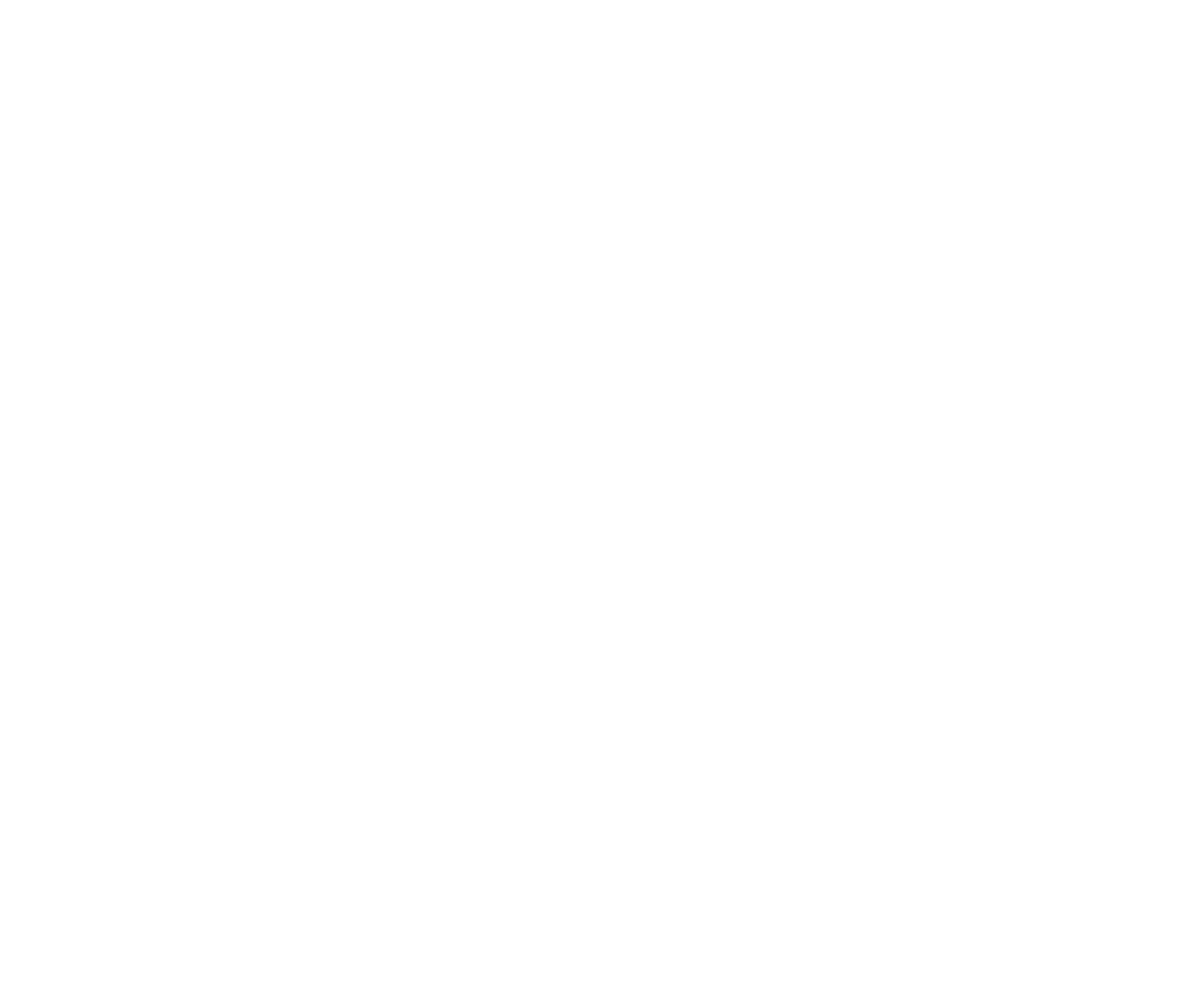

